\title{
An Optical Study to Estimate Orientational Order Parameter on Some Benzylideneaminophenylbenzoate Liquid Crystals
}

\author{
C.M. Subhan ${ }^{a}$, R. Jeevan Kumar ${ }^{a}$, P. PAnduranga ${ }^{a}$, B. Jayashree ${ }^{b}$ \\ AND K. FAKRUDDIN ${ }^{c, *}$ \\ ${ }^{a}$ Department of Physics, Sri Krishnadevaraya University, Anantapur, India \\ ${ }^{b}$ Department of Physics, Maharani's Science College, Bangalore, India \\ ${ }^{c}$ Department of Physics, Ghousia College of Engineering, Ramanagaram, India
}

(Received October 24, 2014; in final form August 23, 2015)

\begin{abstract}
The birefringence technique has been used for the evaluation of order parameter in the nematic phase along with the density measurements, which are needed for the calculation of molecular polarizabilities of the compounds. Birefringence and density studies have been carried out on the three liquid crystalline compounds viz.

(1) (E)-4-((4-hexyloxy)benzylidene)amino)phenyl 4-butoxybenzoate

(2) (E)-4-((4-hexyloxy)benzylidene)amino)phenyl 4-(decycloxy)benzoate and

(3) (E)-4-((4-hexyloxy)benzylidene)amino)phenyl 4-(tridecycloxy)benzoate.

Using birefringence and density data the molecular polarizabilities are evaluated by two different internal field models due to Vuks and Neugebauer. The mean molecular polarizability of the compounds is also evaluated by employing modified Lippincott $\delta$-function model. The results reveal that both the theoretical and experimental values obtained are very near to each other.
\end{abstract}

DOI: 10.12693/APhysPolA.129.284

PACS/topics: 42.79.Kr, 78.15.+e, 61.30.Gd

\section{Introduction}

Liquid crystalline material research has been contributed both to the development of liquid crystal display technology and also to the better understanding of phase behavior for soft condensed matter system. Thus the knowledge of physical properties is necessary. The attempts are continuously being made to study the material properties of compounds for better insight into the basic understanding of liquid crystalline behavior, so that a newer and more acceptable class of materials suitable for display devices is to emerge.

The orientational order parameter is considered to be one of the most important material parameter of the nematic phase, which determines all of its anisotropic properties and the relations between macroscopic and microscopic properties [1, 2].

In the present work, the birefringence and dilatometry techniques [3, 4] are employed for the determination of refractive indices and densities at various temperatures. By using the refractive indices and density data, molecular polarizabilities and order parameters were estimated for the compounds viz.,

comp. 1: (E)-4-((4-(hexyloxy)benzylidene)amino)phenyl 4-butoxy benzoate

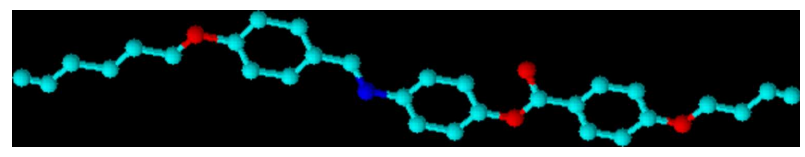

*corresponding author; e-mail: khaji_fakruddin@rediffmail.com melting temperature $110^{\circ} \mathrm{C}$, clearing temperature $244.9^{\circ} \mathrm{C}$

comp. 2: $(E)-4-((4-($ hexyloxy)benzylidene)amino)phenyl 4-(decycloxy)benzoate

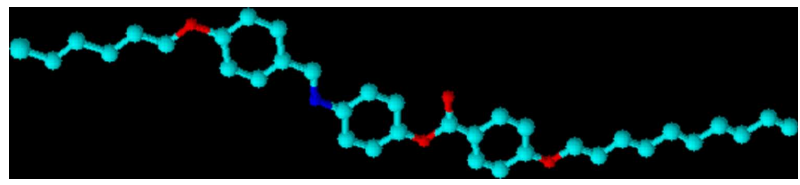

melting temperature $106^{\circ} \mathrm{C}$, clearing temperature $210.5^{\circ} \mathrm{C}$

comp. 3: (E)-4-((4-(hexyloxy)benzylidene)amino)phenyl 4-(tridecycloxy)benzoate

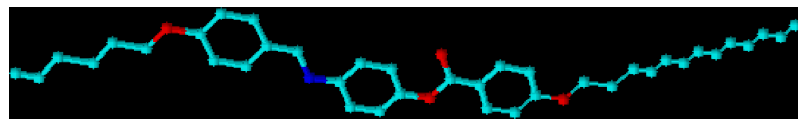

melting temperature $94.9^{\circ} \mathrm{C}$, clearing temperature $199.6^{\circ} \mathrm{C}$.

\section{Experimental}

A polarizing microscope (SD-Techs)/1510 with hot stage is used to measure the transition temperatures. The substance is sandwiched between a glass plate and cover slip which is placed in heating block along with thermometer. The temperature of the hot stage is controlled by varying the voltage of the variac. The temperature is measured by thermocouple sensor which is placed in the heating block.

The refractive indices of the liquid crystalline compounds were measured with wedge shaped glass cell and modified spectrometer $[5,6]$. A wedge shaped glass cell was prepared with two optical flat rectangular glass 

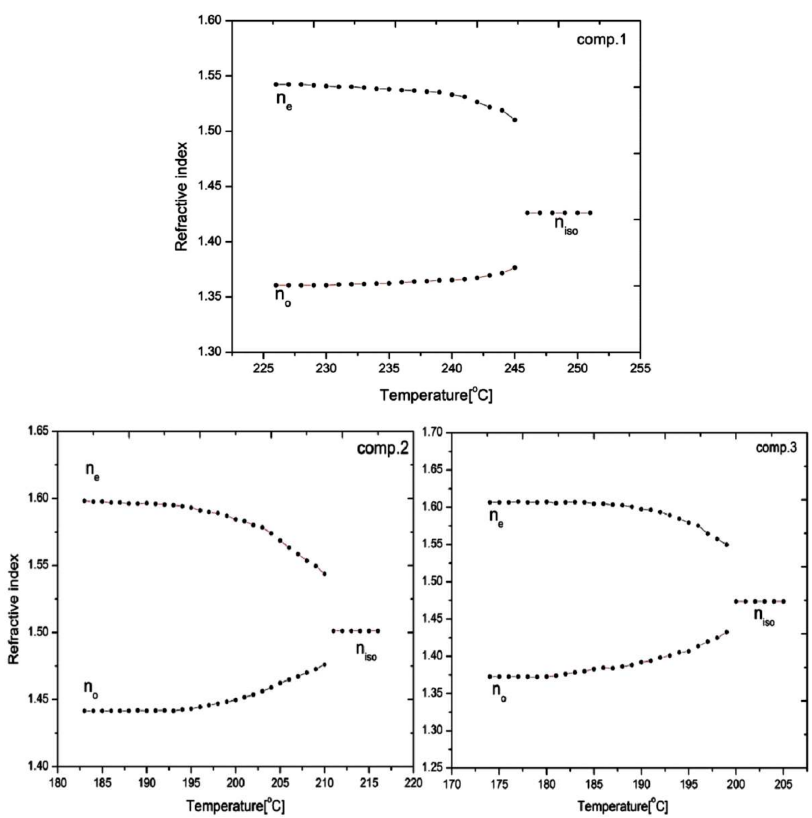

Fig. 1. Temperature variation of refractive index.

plates $(50 \mathrm{~mm} \times 25 \mathrm{~mm})$ sandwiched with glass slide of $0.05 \mathrm{~mm}$ thick which acts as a wedge spacer. The cell is filled with the liquid crystal material. The liquid crystal material in the cell acts as a uniaxial crystal with its optic axis parallel to the edge of the spacer glass plate. The accuracy in the measured refractive indices was \pm 0.0005 .

The U-shaped bi-capillary pyknometer in conjunction with cathetometer was used for the density measurements at various temperatures. The absolute error of the measurement of the densities is $\pm 10^{-4} \mathrm{~g} / \mathrm{cm}^{3}$. The cooling rate during the measurement was $0.5 \mathrm{~K} / \mathrm{h}$.

For the estimation of the molecular polarizabilities of liquid crystalline compounds, the authors have considered the Vuks and Neugebauer models. The relevant equations of the two models are given below.

\subsection{Vuks method}

Recently Pardhasaradhi et al. [7] applied this method to estimate molecular polarizabilities of liquid crystalline molecules and the equations are as follows:

$$
\begin{aligned}
& \alpha_{\mathrm{e}}=\left[\frac{3}{4 \pi N}\right] \frac{n_{\mathrm{e}}^{2}-1}{\bar{n}^{2}-1}, \\
& \alpha_{\mathrm{o}}=\left[\frac{3}{4 \pi N}\right] \frac{n_{\mathrm{o}}^{2}-1}{\bar{n}^{2}-1},
\end{aligned}
$$

where $N$ is the number of molecules per unit volume, $n_{\mathrm{e}}$ and $n_{\mathrm{o}}$ are the extraordinary and ordinary refractive indices of the LC molecule, $\bar{n}^{2}=\frac{n_{\mathrm{e}}^{2}+2 n_{\mathrm{o}}^{2}}{3}$ and $N=N_{\mathrm{A}} \rho / M$, where $N_{\mathrm{A}}$ is the Avogadro number, $\rho$ is the density and $M$ is the molecular weight.

\subsection{Neugebauer method}

Subramanyam et al. [8] applied this method to liquid crystal molecules and recently Lalitha Kumari et al. [9] also used this method. According to this method the molecular polarizabilities are

$$
\begin{aligned}
& \alpha_{\mathrm{e}}=A B-3 \pm \sqrt{\left.(A B-3)^{2}-4 A B\right) / 2 A}, \\
& \alpha_{\mathrm{o}}=A B+3 \pm \sqrt{\left.(A B+3)^{2}-16 A B\right) / 4 A},
\end{aligned}
$$

where

$$
A=\left[\frac{4 \pi N}{3}\right]\left[\frac{n_{\mathrm{e}}^{2}+2}{n_{\mathrm{e}}^{2}-1}\right]+\left[\frac{2\left(n_{\mathrm{o}}^{2}+2\right)}{n_{\mathrm{o}}^{2}-1}\right]
$$

and

$$
B=9\left(\bar{n}^{2}-1\right) /\left[\left(4 \pi N_{i}\right)\left(\bar{n}^{2}+2\right)\right] .
$$

$N_{i}$ is the number of molecules per unit volume in the isotropic phase.

Using the above two models the molecular polarizabilities and the polarizability anisotropy $\left(\alpha_{\mathrm{e}}-\alpha_{\mathrm{o}}\right)$ of the above liquid crystalline compounds were calculated and illustrated in Tables I, II and III.

TABLE I

Ordinary and extraordinary polarizabilities of comp. 1 in liquid crystalline state $\left[10^{-24} \mathrm{~cm}^{3}\right]$.

\begin{tabular}{c|c|c|c|c|c|c}
\hline \hline$T$ & \multicolumn{3}{|c|}{ Vuks } & \multicolumn{3}{|c}{ Neugebauer } \\
\cline { 2 - 7 }$\left[{ }^{\circ} \mathrm{C}\right]$ & $\alpha_{\mathrm{e}}$ & $\alpha_{\mathrm{o}}$ & $\alpha_{\mathrm{e}}-\alpha_{\mathrm{o}}$ & $\alpha_{\mathrm{e}}$ & $\alpha_{\mathrm{o}}$ & $\alpha_{\mathrm{e}}-\alpha_{\mathrm{o}}$ \\
\hline 245 & 63.063 & 44.045 & 19.018 & 60.970 & 45.020 & 15.950 \\
244 & 64.355 & 43.375 & 20.980 & 62.101 & 44.430 & 17.671 \\
243 & 64.800 & 43.120 & 21.680 & 62.489 & 44.203 & 18.286 \\
242 & 65.494 & 42.813 & 22.681 & 63.097 & 43.939 & 19.158 \\
241 & 66.148 & 42.610 & 23.538 & 63.673 & 43.775 & 19.898 \\
240 & 66.423 & 42.508 & 23.915 & 63.915 & 43.690 & 20.225 \\
239 & 66.747 & 42.450 & 24.297 & 64.202 & 43.650 & 20.552 \\
238 & 66.828 & 42.367 & 24.461 & 64.271 & 43.573 & 20.698 \\
237 & 66.961 & 42.305 & 24.656 & 64.387 & 43.519 & 20.868 \\
236 & 67.039 & 42.231 & 24.808 & 64.454 & 43.451 & 21.003 \\
235 & 67.188 & 42.115 & 25.073 & 64.582 & 43.346 & 21.236 \\
234 & 67.236 & 42.096 & 25.140 & 64.624 & 43.329 & 21.295 \\
233 & 67.385 & 42.034 & 25.351 & 64.755 & 43.277 & 21.478 \\
232 & 67.497 & 41.998 & 25.499 & 64.853 & 43.248 & 21.605 \\
231 & 67.506 & 41.965 & 25.541 & 64.860 & 43.216 & 21.644 \\
230 & 67.618 & 41.876 & 25.742 & 64.956 & 43.135 & 21.821 \\
229 & 67.709 & 41.869 & 25.840 & 65.037 & 43.133 & 21.904 \\
228 & 67.814 & 41.860 & 25.954 & 65.130 & 43.130 & 22.000 \\
227 & 67.814 & 41.860 & 25.954 & 65.130 & 43.130 & 22.000 \\
226 & 67.814 & 41.860 & 25.954 & 65.130 & 43.130 & 22.000
\end{tabular}

\subsection{Estimation of mean polarizability from modified Lippincott $\delta$-function method}

The modified Lippincott $\delta$-function model is found to be effective $[10,11]$ for different types of liquid crystals. In the present study the modified Lippincott $\delta$-function model has been used to estimate the mean polarizability in terms of parallel bond component $\left(\sum \alpha_{\|_{\mathrm{p}}}\right)$ the perpendicular bond component $\left(\sum 2 \alpha_{\perp}\right)$ and the non-bond region electron contribution $\left(\sum \alpha_{\|_{\mathrm{n}}}\right)$. The expression for the mean polarizability is given as

$$
\alpha_{\mathrm{M}}=\frac{1}{3}\left(\sum \alpha_{\|_{\mathrm{p}}}+\sum \alpha_{\|_{\mathrm{n}}}+\sum 2 \alpha_{\perp}\right),
$$


TABLE II

Ordinary and extraordinary polarizabilities of comp. 2 in liquid crystalline state $\left[10^{-24} \mathrm{~cm}^{3}\right]$.

\begin{tabular}{c|c|c|c|c|c|c}
\hline \hline$T$ & \multicolumn{3}{|c|}{ Vuks } & \multicolumn{3}{|c}{ Neugebauer } \\
\cline { 2 - 7 }$\left[{ }^{\circ} \mathrm{C}\right]$ & $\alpha_{\mathrm{e}}$ & $\alpha_{\mathrm{o}}$ & $\alpha_{\mathrm{e}}-\alpha_{\mathrm{o}}$ & $\alpha_{\mathrm{e}}$ & $\alpha_{\mathrm{o}}$ & $\alpha_{\mathrm{e}}-\alpha_{\mathrm{o}}$ \\
\hline 210 & 73.287 & 64.750 & 8.537 & 72.233 & 66.634 & 5.599 \\
209 & 73.969 & 64.265 & 9.704 & 72.933 & 66.110 & 6.823 \\
208 & 74.537 & 63.775 & 10.762 & 73.441 & 65.567 & 7.874 \\
207 & 75.227 & 63.384 & 11.843 & 74.043 & 65.139 & 8.904 \\
206 & 75.911 & 62.918 & 12.993 & 74.678 & 64.695 & 9.983 \\
205 & 76.752 & 62.495 & 14.257 & 75.448 & 64.307 & 11.141 \\
204 & 77.245 & 62.152 & 15.093 & 75.890 & 63.989 & 11.901 \\
203 & 78.104 & 61.713 & 16.391 & 76.659 & 63.594 & 13.065 \\
202 & 79.053 & 61.187 & 17.866 & 77.518 & 63.139 & 14.379 \\
201 & 79.623 & 60.551 & 19.072 & 78.000 & 62.543 & 15.457 \\
200 & 80.695 & 59.874 & 20.821 & 78.930 & 61.934 & 16.996 \\
199 & 81.308 & 59.350 & 21.958 & 79.452 & 61.453 & 17.999 \\
198 & 82.089 & 58.763 & 23.326 & 80.119 & 60.921 & 19.198 \\
197 & 83.035 & 58.242 & 24.793 & 80.936 & 60.463 & 20.473 \\
196 & 83.888 & 57.843 & 26.045 & 81.674 & 60.122 & 21.552 \\
195 & 84.805 & 57.525 & 27.280 & 82.413 & 59.816 & 22.597 \\
194 & 85.733 & 57.188 & 28.545 & 83.219 & 59.541 & 23.678 \\
193 & 86.501 & 56.988 & 29.513 & 83.890 & 59.391 & 24.499 \\
192 & 86.865 & 56.927 & 29.938 & 84.147 & 59.306 & 24.841 \\
191 & 87.173 & 56.881 & 30.292 & 84.417 & 59.280 & 25.137 \\
190 & 87.459 & 56.811 & 30.648 & 84.644 & 59.213 & 25.431 \\
189 & 87.750 & 56.733 & 31.017 & 84.917 & 59.171 & 25.746 \\
188 & 87.761 & 56.642 & 31.119 & 84.921 & 59.083 & 25.838 \\
187 & 87.719 & 56.629 & 31.090 & 84.881 & 59.068 & 25.813 \\
186 & 87.934 & 56.592 & 31.342 & 85.069 & 59.045 & 26.024 \\
185 & 88.026 & 56.563 & 31.463 & 85.148 & 59.022 & 26.126 \\
184 & 87.869 & 56.561 & 31.308 & 85.007 & 59.011 & 25.996
\end{tabular}

$$
\begin{aligned}
& \sum \alpha_{\|_{\mathrm{p}}}=\frac{4 n A}{a_{0}} \exp \left(\frac{\left(T-T_{\mathrm{c}}\right)}{T_{\mathrm{c}}}\right)\left(\frac{R^{2}}{4}+\frac{1}{2 C_{R^{2}}}\right) \\
& \quad \times \exp \left(-\frac{\left(x_{\mathrm{A}}-x_{\mathrm{B}}\right)^{2}}{4}\right) .
\end{aligned}
$$

Here $n$ is the bond order, $A$ - the $\delta$-function strength, $T$ is the temperature in the liquid crystalline phase, $T_{C}$ is the clearing temperature, $R$ is the bond length, $X_{\mathrm{A}}$ and $X_{B}$ are the Pauling electronegativities of atoms $\mathrm{A}$ and $\mathrm{B}$ in the bond $\mathrm{AB}, a_{0}$ is the radius of the first Bohr orbit of the atomic hydrogen, and $C_{R}$ is the geometric mean molecular $\delta$-function strength. For the second term on the right hand side of Eq. (5) we have

$$
\sum \alpha_{\|_{\mathrm{n}}}=\sum_{j} f_{j} \alpha_{j}
$$

Here $f_{j}$ is the fraction of the non-bonded electrons of the $j$-th atom and $\alpha_{j}$ is its atomic polarizability.

For the third term in Eq. (5):

$$
\sum 2 \alpha_{\perp}=n_{\mathrm{df}} \frac{\sum x_{j}^{2} \alpha_{j}}{\sum x_{j}^{2}} .
$$

Here $n_{\mathrm{df}}$ is the number of degrees of freedom given by
TABLE III

Ordinary and extraordinary polarizabilities of comp. 3 in liquid crystalline state $\left[10^{-24} \mathrm{~cm}^{3}\right]$.

\begin{tabular}{c|c|c|c|c|c|c}
\hline \hline$T$ & \multicolumn{3}{|c|}{ Vuks } & \multicolumn{3}{|c}{ Neugebauer } \\
\cline { 2 - 7 }$\left[{ }^{\circ} \mathrm{C}\right]$ & $\alpha_{\mathrm{e}}$ & $\alpha_{\mathrm{o}}$ & $\alpha_{\mathrm{e}}-\alpha_{\mathrm{o}}$ & $\alpha_{\mathrm{e}}$ & $\alpha_{\mathrm{o}}$ & $\alpha_{\mathrm{e}}-\alpha_{\mathrm{o}}$ \\
\hline 199 & 83.306 & 62.530 & 20.776 & 80.706 & 63.830 & 16.876 \\
198 & 84.804 & 61.302 & 23.502 & 81.982 & 62.713 & 19.269 \\
197 & 86.152 & 60.430 & 25.722 & 83.137 & 61.937 & 21.200 \\
196 & 88.129 & 59.402 & 28.727 & 84.839 & 61.047 & 23.792 \\
195 & 89.079 & 58.334 & 30.745 & 85.624 & 60.061 & 25.563 \\
194 & 89.970 & 58.035 & 31.935 & 86.398 & 59.821 & 26.577 \\
193 & 90.877 & 57.346 & 33.531 & 87.164 & 59.202 & 27.962 \\
192 & 91.699 & 56.886 & 34.813 & 87.868 & 58.801 & 29.067 \\
191 & 92.344 & 56.222 & 36.122 & 88.405 & 58.191 & 30.214 \\
190 & 92.545 & 55.917 & 36.628 & 88.567 & 57.906 & 30.661 \\
189 & 93.243 & 55.351 & 37.892 & 89.156 & 57.395 & 31.761 \\
188 & 93.614 & 55.016 & 38.598 & 89.468 & 57.089 & 32.379 \\
187 & 93.811 & 54.663 & 39.148 & 89.625 & 56.756 & 32.869 \\
186 & 93.991 & 54.699 & 39.292 & 89.785 & 56.802 & 32.983 \\
185 & 94.009 & 54.478 & 39.531 & 89.790 & 56.587 & 33.203 \\
184 & 94.483 & 54.000 & 40.483 & 90.188 & 56.148 & 34.040 \\
183 & 94.566 & 53.759 & 40.807 & 90.250 & 55.917 & 34.333 \\
182 & 94.579 & 53.488 & 41.091 & 90.249 & 55.653 & 34.596 \\
181 & 94.451 & 53.146 & 41.305 & 90.121 & 55.311 & 34.810 \\
180 & 94.773 & 52.917 & 41.856 & 90.395 & 55.106 & 35.289 \\
179 & 94.681 & 52.860 & 41.821 & 90.311 & 55.045 & 35.266 \\
178 & 94.625 & 52.882 & 41.743 & 90.261 & 55.064 & 35.197 \\
177 & 94.739 & 52.873 & 41.866 & 90.360 & 55.062 & 35.298 \\
176 & 94.522 & 52.844 & 41.678 & 90.163 & 55.023 & 35.140 \\
175 & 94.497 & 52.830 & 41.667 & 90.140 & 55.009 & 35.131 \\
174 & 94.473 & 52.816 & 41.657 & 90.117 & 54.994 & 35.123
\end{tabular}

the equation

$$
n_{\mathrm{df}}=3 N-2 n_{\mathrm{b}}
$$

where $N$ is the number of atoms and $n_{\mathrm{b}}$ is the number of bonds in the molecule.

\subsection{Estimation of orientational order parameter in the nematic phase}

In the Vuks method the order parameter is given by $[7,12]$ :

$$
S=\left(\frac{\alpha}{\alpha_{\|}-\alpha_{\perp}}\right) \frac{n_{\mathrm{e}}^{2}-n_{\mathrm{o}}^{2}}{\bar{n}^{2}-1},
$$

where

$$
\bar{n}^{2}=\left(\frac{n_{\mathrm{e}}^{2}+2 n_{\mathrm{o}}^{2}}{3}\right) .
$$

The order parameter in case of the Neugebauer method [13]:

$$
S=\left(\frac{\alpha}{\alpha_{\|}-\alpha_{\perp}}\right) f(B),
$$

where

$$
f(B)=\frac{9}{4 A B}\left[\left(B^{2}-\frac{10}{3} B+1\right)^{1 / 2}+\left(\frac{B}{3}\right)-1\right]
$$


and

$$
B=\frac{n^{2}-1}{n^{2}+1}\left[\frac{n_{\mathrm{e}}^{2}+2}{n_{\mathrm{e}}^{2}-1}+\frac{2\left(n_{\mathrm{o}}^{2}+2\right)}{n_{\mathrm{o}}^{2}-1}\right] .
$$

\section{Results and discussion}

In the present investigation the refractive indices of the benzylidene amino phenyl benzoate liquid crystalline compounds with alkoxy chain length $n=4,10,12$ and $m=6$ are measured using modified spectrometer with small angled prism which houses the crystalline compound. The small angled prism is placed in a heating block for measurement of refractive indices with temperature and a monochromatic source of wavelength $589.3 \mathrm{~nm}$ is used. During isotropic-nematic phase transformation the incident light splits into two one higher and the other lower than the isotropic value called extraordinary $n_{\mathrm{e}}$ and ordinary $n_{\mathrm{o}}$ refractive indices. In the nematic region the value of $n_{\mathrm{e}}$ increases where as the value of $n_{\mathrm{o}}$ decreases with temperature and both attains saturation deep in nematic region. The variation of refractive indices with temperature in isotropic and nematic region is illustrated in Fig. 1.

The temperature variation of density is measured by dilatometer attached with U-shape bicapillary pyknometer and represented in Fig. 2. It is found that with rise in temperature density decreases and at phase transformations there are density jumps.
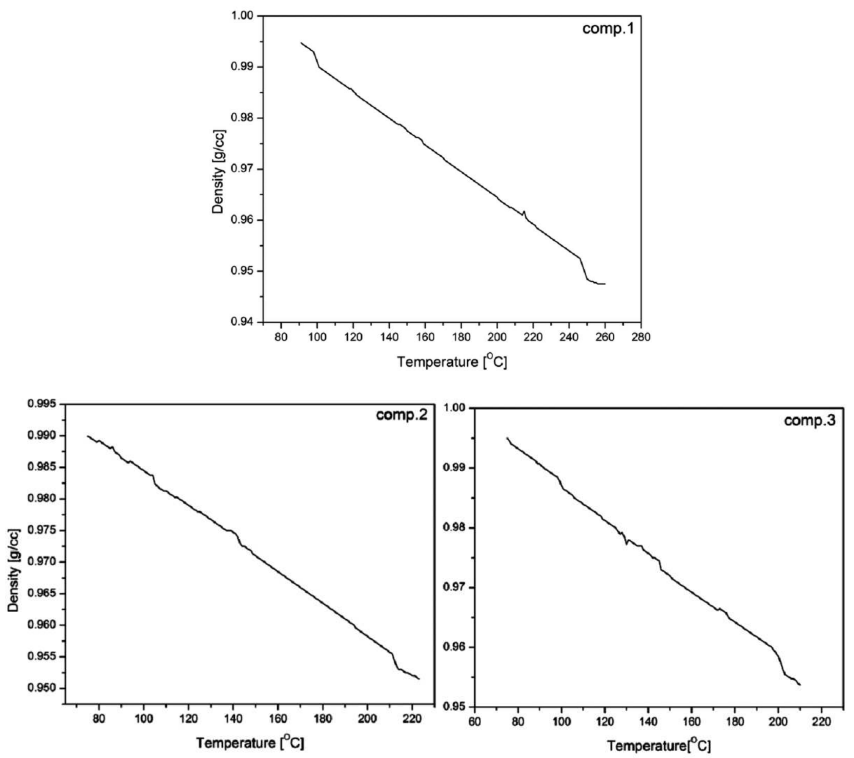

Fig. 2. Temperature variation of density.

The molecular polarizabilities are estimated by Vuks and Neugebauer internal field models and their molecular anisotropies are presented in Tables I, II and III. Further the mean molecular polarizability is evaluated by modified Lippincott $\delta$-function model and the values are compared with Vuks and Neugebauer models and the results are shown in Table IV. The order parameter is evaluated for both Vuks and Nuegauber model and shown in Fig. 3.
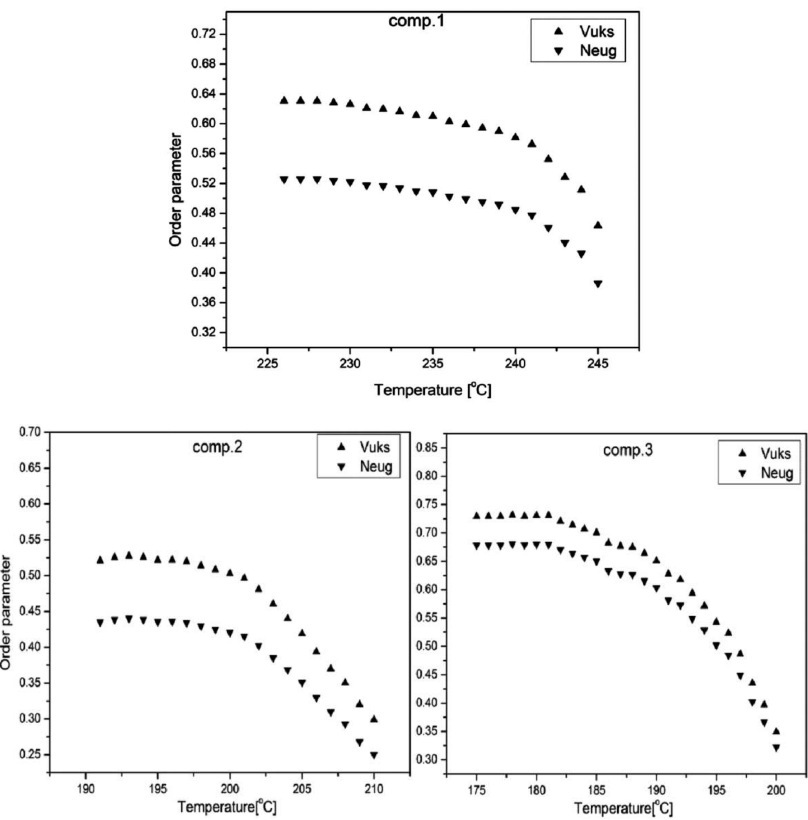

Fig. 3. Variation of order parameter with temperature. TABLE IV

Mean molecular polarizabilities obtained in the liquid crystalline compounds by different methods $\left[10^{-24} \mathrm{~cm}^{3}\right]$

\begin{tabular}{c|c|c|c|c|c|c}
\hline \hline meth. & \multicolumn{3}{|c|}{ Lippincott $\delta$-function } & Vuks & Neugebauer \\
\hline comp. & $\alpha_{\|}$ & $\alpha_{\|_{\mathrm{n}}}$ & $2 \alpha_{\perp}$ & \multicolumn{3}{|c}{$\alpha_{\mathrm{M}}$} \\
\hline 1 & 94.79 & 1.87 & 52.34 & 49.67 & 50.42 & 50.46 \\
2 & 123.94 & 1.87 & 64.24 & 63.35 & 67.77 & 67.02 \\
3 & 129.33 & 1.87 & 70.95 & 67.38 & 67.79 & 67.79
\end{tabular}

\section{Conclusions}

1. The birefringence observed in the compounds is around 0.15 to 0.23 ;

2. The ordinary and extraordinary refractive indices attain saturation deep in the nematic phase;

3. The mean molecular polarizabilities obtained from birefringence data using the internal field models due to Vuks and Neugebauer are in reasonably good agreement with modified Lippincott $\delta$ function model;

4. The orientational order parameter evaluated using both the models shows decrease with increase of temperature;

5. The orientational order parameter estimated by the Vuks and Neugebauer method is in between 0.3 to 0.7 which is in accordance with literature data available;

6. The orientational order parameter value evaluated using the Neugebauer method is always low when compared to other methods. The same results are obtained in our investigations. 


\section{Acknowledgments}

Authors express their thanks to the Department of Physics, S.K. University, Anantapur, for providing laboratory facilities. The authors are thankful to T.N. Swamy, Senior Scientist, CLRI, Chennai for providing liquid crystalline compounds to carry out physical properties. The financial support rendered by the UGC under SAP [No.F.530/8/DRS/2010 (SAP-I)] and Department of Science and Technology under FIST [SR/FST/PSI116/2007], New Delhi, are gratefully acknowledged. The authors are also thankful to Department of Physics of Ghousia College of Engineering, Ramanagaram for providing laboratory facilities. The financial support rendered by VTU Belgaum under VTU research grant scheme for the research project [No. VTU/Aca/2011$12 / \mathrm{A}-9 / 750]$ is gratefully acknowledged. One of the author C.M. Subhan is thankful to UGC New Delhi for providing BSR fellowship.

\section{References}

[1] W.H. De Jeu, Physical Properties of Liquid Crystalline Materials, Gordon and Breach, New York 1980.

[2] W. Kuczynski, B. Zywuchki, J. Matecki, Mol. Cryst. Liq. Cryst. 381, 1 (2002).
[3] S.K. Pal, V.A. Raghunathan, S. Kumar, Liq. Cryst. 34, 135 (2007).

[4] C.L. Toh, J. Xu, C. He, Liq. Cryst. 35, 241 (2008).

[5] I. Haller, H.A. Huggins, H.R. Lilienthal, T.R. McGuire, J. Phys. Chem. 77, 950 (1973).

[6] D. Venkata Rao, P. Pardhasaradhi, V.G.K.M. Pisipati, P.V. Datta Prasad, Phase Transit. 88, 137 (2015).

[7] P. Pardhasaradhi, P.V. Datta Prasad, D. Madhavi Latha, V.G.K.M. Pisipati, G. Padmaja Rani, Phase Transit. 85, 1031 (2012).

[8] H.S. Subramhanyam, D. Krishnamurti, Mol. Cryst. Liq. Cryst. 22, 239 (1973)

[9] J. Lalitha Kumari, P.V. Datta Prasad, D. Madhavi Latha, V.G.K.M. Pisipati, Phase Transit. 85, 52 (2012).

[10] K. Fakruddin, R. Jeevan Kumar, P.V. Datta Prasad, V.G.K.M. Pisipati, Mol. Cryst. Liq. Cryst. 511, 146 (2009).

[11] V.R. Murthy, S.V. Naidu, R.N.V. Ranga Reddy, Mol. Cryst. Liq. Cryst. 59, 27 (1980).

[12] P. Adamski, A. Dylik-Gromisc, Mol. Cryst. Liq. Cryst. 35, 171 (1976)

[13] I. Haller, H.A. Huggins, M.J. Freisner, Mol. Cryst. Liq. Cryst. 16, 53 (1972). 